\title{
A Comparison of Techniques to Optimize Measurement of Voltage Changes in Electrical Impedance Tomography by Minimizing Phase Shift Errors
}

\author{
A. J. Fitzgerald, D. S. Holder*, L. Eadie, C. Hare, and R. H. Bayford
}

\begin{abstract}
In electrical impedance tomography, errors due to stray capacitance may be reduced by optimization of the reference phase of the demodulator. Two possible methods, maximization of the demodulator output and minimization of reciprocity error have been assessed, applied to each electrode combination individually, or to all combinations as a whole. Using an EIT system with a single impedance measuring circuit and multiplexer to address the $\mathbf{1 6}$ electrodes, the methods were tested on resistor-capacitor networks, saline-filled tanks and humans during variation of the saline concentration of a constant fluid volume in the stomach. Optimization of each channel individually gave less error, particularly on humans, and maximization of the output of the demodulator was more robust. This method is, therefore, recommended to optimize systems and reduce systematic errors with similar EIT systems.
\end{abstract}

Index Terms-Demodulator, EIT, reciprocity error.

\section{INTRODUCTION}

$\mathbf{E}$ LECTRICAL impedance tomography (EIT), a medical imaging method that maps the distribution of conductivity in the body, requires precise measurements of the surface voltages that result from applied currents. The combined effects of common mode voltages, stray capacitance and electrode-skin contact impedance contribute to errors that affect the measured voltages. In this paper, several methods of correction for these errors are investigated and compared.

\section{A. Sources of Error in EIT}

EIT is an imaging technique that uses alternating electrical currents in the range of $10 \mathrm{kHz}$ to $1 \mathrm{MHz}$ to image the electrical conductivity of the body [1]. Recent EIT systems may apply current or voltage, and measure in-phase and quadrature components. This work used a system based on the Sheffield Mark

Manuscript received August 31, 2001; revised February 13, 2002. Asterisk indicates corresponding author.

A. J. Fitzgerald was with University College London (UCL), Middlesex Hospital, London W1N 8AA, U.K. He is now with the Department of Medical Physics, Leeds University, Wellcome Wing, Leeds General Infirmary, Leeds LS1 3EX, U.K.

*D. S. Holder is with the Department of Clinical Neurophysiology University College London, Middlesex Hospital, London W1N 8AA, U.K.

L. Eadie and R. H. Bayford are with the Department of Clinical Neurophysiology University College London, Middlesex Hospital, London W1N 8AA, U.K.

C. Hare is with the Department of Radiology, University College London, Middlesex Hospital, London, W1N 8AA U.K.

Publisher Item Identifier 10.1109/TMI.2002.800577.
1 APT system [see [1]], which makes serial in-phase voltage measurements, and is only used for dynamic images. A constant current generator is connected to electrodes on the body, usually via a multiplexer, and supplies current to the object to be imaged. The resulting sinusoidally varying voltage on the body is sampled on the surface at other electrodes and differentially amplified. The signal can be demodulated, by rectification using the reference waveform, and filtered, to obtain a voltage level that is a measure of the impedance.

The principal sources of errors in the front end arise from common mode effects, skin electrode contact impedance and stray capacitances [2]-[5]. The final error for each measurement is dependent on the complex interaction of these effects, and will differ for each electrode combination [6].

\section{B. Explanation of Optimization Methods}

It is desirable to correct for the errors introduced by the various sources. A number of approaches have been used. One is to perform a calibration on components of known value, such as a resistor or resistor-capacitor network [7]. The problem with this technique is that the performance and errors of a system depend on the load impedance and the presence of skin-electrode contact impedance [7], [8]. A true calibration can only be performed under conditions identical to imaging, a situation which cannot be achieved in practice since the effects of skin electrode contact impedance at the time of imaging are difficult to reproduce in a model.

Two principal methods for optimization are:

1) Maximization of the Voltage Output From the Demodulator: A demodulator (or lock-in amplifier) is used to convert the sinusoidal recorded signal to a dc level which depends on the phase of the signal. The phase at which the sine wave is measured is determined by a reference signal. In practice, demodulation is usually achieved by a digital process, in which the sine wave is sampled once each cycle at the phase set by the reference signal, or by rectification and low-pass filtering, with the phase of the rectification set by the reference signal.

In practice, the voltage recorded by an EIT system contains resistive and reactive components from the test object, and also errors, which are largely due to stray capacitance. The errors may, therefore, be reduced by setting the demodulator to record in-phase (Fig. 1). Unfortunately, in practice, it is not possible to know exactly what this phase setting is, because the applied 
Applied current

Voltages :

A) Resistance

Measure in-phase

B) Capacitance

Measure out-of-phase

C) Capacitance

Measure in-phase

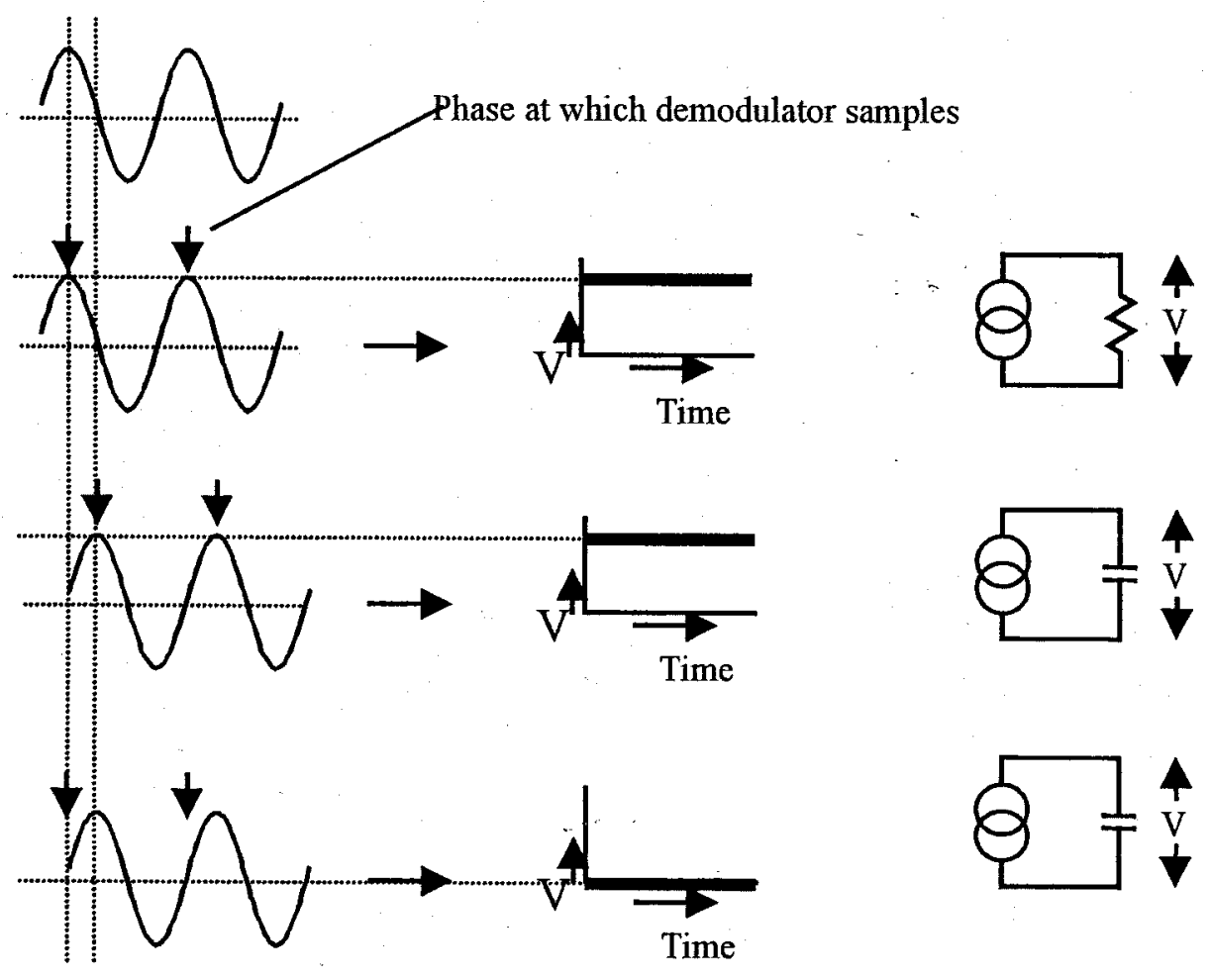

Current/voltage
Demodulated voltage

Fig. 1. Demodulation. The voltage that results from an applied current is in phase for a resistor (A) and $90^{\circ}$ out of phase for a capacitor (B\&C). The in or out of phase components may be selected by setting the phase at which the demodulator measures. The effects of capacitance may be ignored by setting the demodulator to measure at in-phase; it will then only transmit the resistive component of a signal.

current will itself have an altered phase because of stray capacitance and electrode impedance. The principle of the "optimized demodulator" method is to assume that the object imaged is resistive; the measuring phase of the demodulator is then set to that which yields the largest signal. This assumes that the impedance itself is dominated by the in phase component, since this is affected less by errors than the quadrature component. At frequencies less than $50 \mathrm{kHz}$, this assumption is reasonable because the magnitude of the reactive component of human tissue is less than $10 \%$ of the real component [9].

2) Minimization of Reciprocity Error (RE): An ideal impedance measuring circuit that injects current through port $\mathrm{AB}$ on an arbitrary region of conductivity will sense a voltage, $V_{1}$, at port CD. If the current and voltage sensing ports are reversed, a voltage $V_{2}$ will be sensed at $\mathrm{AB}$. Reciprocity theory states that $V_{2}$ will be identical to $V_{1}[10]$. In practice, $V_{1}$ and $V_{2}$ will differ by an amount that depends on the errors present and the phase of the reference at the demodulator. The RE may be defined as

$$
\mathrm{RE}=\frac{2 \cdot(V 1-V 2)}{(V 1+V 2)}
$$

Minimization of the RE has been employed in the widely used Sheffield Mark 1 system [1], [11], as it might be expected to give a phase offset for demodulator measurement which approximates to the in-phase component of voltage, because this component is less affected by errors than the quadrature component.
3) Optimization for Individual or all Electrode Combinations: The method of optimization used with the Sheffield mark 1 system [11] is to set the phase of the reference waveform in hardware, so that the largest REs in the measurement set on the object are minimized. This method of setting a single phase offset for all electrode combinations may not be optimal because errors vary from channel to channel, but it has the advantages of simplicity and speed. For each of the above two methods, the phase or reciprocity could be optimized individually for each electrode combination, or an average or minimum value could be determined for all electrodes. Methods for optimization for both individual or all electrode combinations were tested.

\section{Purpose}

The purpose of this work was to assess the above two types of methods of optimization empirically under different experimental conditions. The methods were compared for a single phase that was used for all the electrode combinations, or individual phases determined for each combination.

\section{Experimental Design}

Four optimization techniques (Section II-B) were tested in three different situations, using a 16-electrode EIT system built in our laboratory:

1) The Cardiff Cole Phantom [12]: This is a rim and wheel type resistor-capacitor phantom which presents identical impedance distributions to each drive configuration. The 
phantom has the facility to model skin impedance, although, unlike in in vivo measurements, the modeled contact impedance is identical at each electrode. Errors in the impedance distribution of the phantom from component tolerances are less than $0.3 \%$ [13]. It is not possible to introduce local impedance changes but the phantom has circular symmetry so that errors between different electrode combinations can be compared and used as an index of the accuracy of an optimization method.

2) Saline-Filled Tanks: These represent an intermediate step to in vivo imaging. Saline concentrations were used which gave transfer impedances similar to those encountered with human imaging. The advantage of using a tank was that known resistivity changes could be introduced.

3) Human Studies: The optimization techniques were also investigated directly on humans, as this includes real-life skin/electrode interface impedance. In order to produce a well-defined test situation, saline fluids of different concentrations were syringed into and out of the stomach of volunteers using a naso-gastric tube. The stomach was filled with the same volume of fluid each time so that boundary shape and internal resistivity distribution were unchanged from image to image.

\section{METHODS}

\section{A. EIT Hardware}

An EIT system constructed in this laboratory, the "UCLH EIT Mark Ia system" [14], [15] was used. It can apply frequencies from $225 \mathrm{~Hz}$ to $80 \mathrm{kHz}$, and is optimized for imaging brain function in ambulant patients. A mainbox contains the control hardware and digital signals are passed from it to the headbox, which houses the current source and voltage amplifier circuits, keeping them close to the patient to reduce sources of stray capacitance. It uses 16 electrodes, and all parameters can be set in software. Images are reconstructed on a PC; in this study, they were reconstructed using a back projection algorithm, as in the Sheffield Mark 1 system [11]. The modifications made for brain imaging are the ability to record down to low frequencies, as this may be optimal for some cerebral changes such as epilepsy, and the use of a small headbox on a 10-meter-long lead, so that recordings may be made in ambulant patients, over days, for example when attempting to record seizures.

The system uses a single ended floating current source. Two cascaded programmable gain amplifiers (PGA) provide a software selectable gain range from 1 to 8000 . It uses phase-sensitive coherent analogue demodulation. The reference sine wave phase offset can be set to any integer index from 0 to 255, giving a phase resolution of $1.4^{\circ}$. The phase offset for each channel is downloaded from an ASCII file on the PC to the microcontroller in the headbox, together with the gain value. The frequency was set to $38 \mathrm{kHz}$ for these studies.

A MT8816 crosspoint switch (Mitel Semiconductor, San Diego, CA) is used to multiplex the current to the drive electrodes and connect detection electrodes to the voltage measurement circuit. Using the multiplexer, any combination of two electrodes can deliver current and any other two electrodes can sense voltage. The electrode combinations are downloaded from an ASCII protocol to the microcontroller in a similar manner to phase and gain. The adjacent protocol developed by the Sheffield group [11] was used for the collection of voltage data sets in this study. With this protocol, current is applied to an adjacent pair of electrodes, and voltage is recorded sequentially from all other available adjacent pairs. This is repeated for all electrodes.

\section{B. Optimization Techniques}

For each of the phase optimization techniques, two sets of phases were determined prior to collecting voltage data. One set of phases was determined for each individual electrode combination, and the other was a fixed phase used for all electrode combinations.

\section{1) Maximization of Demodulated Signal:}

Method la: The optimized phase offset for each electrode combination was determined with the UCLH EIT device connected to electrodes on the object to be imaged. First the optimum gain was determined for each electrode combination and then the phase. With the PGA on the record side set to the optimum gain value, the index of the reference signal for coherent demodulation was cycled from 0 through to 255 in 16 steps. The demodulated signal is a sinusoidal function with phase offset. The phase offset at which the maximum occurs was taken to be the optimized phase for that combination of drive and receive electrodes. This was repeated for all drive and receive combinations in the adjacent protocol. In this way, an optimized phase was determined for each position.

Method 1b: A single fixed phase to be used for all electrode combinations was calculated by averaging the optimum phase offsets from all the electrode combinations from method 1a above.

2) $R E$ :

Method 2a: The RE was calculated from voltage measurements at each set of drive and receive combinations with the UCLH EIT device connected to the object to be imaged. The voltage was recorded for every electrode combination in the adjacent protocol, together with its reciprocal, at thirty two phases spanning the range of indices from 0 to 255 . For each combination the RE was calculated at each phase offset using (1). A fourth order polynomial was numerically fitted to the curve of RE against phase. The phase at which RE was a minimum was taken to be the optimized phase for that combination. This was repeated for all positions in the adjacent protocol to give a collection of phases that represented the optimized phase shifts from the reciprocity technique.

Method 2b: A single phase for all the electrode combinations was determined by averaging the REs for all positions at each phase to give a curve of averaged RE versus phase. The phase that minimized average RE was used as the fixed phase for all combinations.

\section{Change in Voltage as a Function of Reference Phase}

The function of measured change in voltage with reference phase was determined using a resistor-capacitor network. A $21.9 \Omega$ resistor in series with a $2.0 \Omega$ reactance (at $38 \mathrm{kHz}$ ), measured with a Hewlett-Packard 4284A impedance analyzer, modeled body impedance. Electrode contact impedance was modeled on each drive and receive channel with a $220 \Omega$ resistor in series with a 10-nF capacitor, after [16]. 
Optimized phases were determined for this circuit using the methods that maximized the demodulator output and minimized RE. Voltage across the load section of the circuit was measured at each phase index from 0 to 255 . A second resistor of value $2.2 \Omega$ was included in series to model a change in impedance of $10.0 \%$, measured on the Hewlett-Packard 4284A. A set of voltages for the range of phase indices from 0 to 255 was recorded on this modified circuit. Percentage change in measured voltage was calculated at each phase index by comparison of the voltage sets for the two circuits.

To investigate the effect of large component mismatches on $\mathrm{RE}$, the same experiment was repeated with the $220 \Omega$ resistor in series with a 10-nF capacitor only on the current drive circuit and not on the voltage measurement channels.

\section{Phantoms}

1) Cardiff Cole Phantom: The Cardiff Cole phantom was set to its purely resistive state, with no contact impedance. Optimized phase values were determined for each of the four techniques at each of 104 electrode combinations for the adjacent protocol. Voltage data sets were then recorded for the optimized phase settings for each technique. The accuracy of different optimization methods was assessed by comparing the voltage changes on introduction of an increased transfer resistance of $5.6 \%$. For each technique, the voltage change was calculated as the average for all 16 drive electrode pair positions, for each of the 13 recording electrode pair positions relative to their drive pair. Analysis of variance (ANOVA) was performed on the log standard deviation of the root mean square residual differences between the expected and measured percentage changes in voltage over the 13 relative recording pair positions.

\section{2) Saline Tank:}

Changes to Background Resistivity: The UCLH EIT device was connected to a cylindrical tank, $200 \mathrm{~mm}$ in diameter, filled with $500 \mathrm{ml}$ of $0.2 \%$ sodium chloride solution at room temperature, using 16 stainless-steel electrodes. Phase shifts and voltages for each of the four optimization techniques were determined and a voltage data set recorded. $10 \%$ of the solution was removed and replaced with distilled water and a second set of voltages was recorded. Replacement of $10 \%$ of the original $0.2 \%$ saline corresponded to an increase in resistivity of $10.7 \%$. Changes of voltage were combined in the same manner as those from the Cardiff phantom.

Resolution of Two Objects in the Tank: Phase shifts and voltage data sets for each of the optimization techniques were determined on the tank filled to a height of $20 \mathrm{~mm}$ with $0.2 \%$ sodium chloride solution at room temperature. Reference sets were recorded for the techniques, each an average of 100 measurements. Two cylindrical polyurethane sponges [17] (Grade $30 \mathrm{H}, 3 \%$ w/v, British Vitafoam Limited, Manchester, U.K.), of diameter $20 \mathrm{~mm}$, height $18 \mathrm{~mm}$ were placed in the tank, each $25 \mathrm{~mm}$ from the center, giving a separation of $50 \mathrm{~mm}$, aligned on a diameter of the tank. Voltage data sets were recorded for each of the techniques at this separation and at six further separations in steps of $2 \mathrm{~mm}$ along the central axis.

Cross-sectional profiles through the central axis that included the sponge positions were determined from the images at the seven separations for each of the optimization techniques. Resolution of the two sponges was expressed as the average of the impedance of the peak changes due to the two sponges, divided by the impedance of the trough between them, expressed as a percentage increase.

3) Human Imaging: In each of three healthy volunteers, 16 ECG electrodes were placed at equal intervals in a horizontal ring on degreased and abraded skin $5 \mathrm{~cm}$ above the umbilicus. The UCLH EIT device was connected to the volunteer and optimized phase values for each technique were determined with the subject in a seated position. The stomach was filled with $250 \mathrm{ml}$ of distilled water at $37^{\circ} \mathrm{C}$ using a naso-gastric tube and voltage data sets were recorded for each of the techniques. The distilled water was syringed out from the stomach and replaced with $0.2 \%$ sodium chloride solution at $37^{\circ} \mathrm{C}$ and voltage data sets were recorded. This was replaced by $0.9 \%$ sodium chloride solution at $37^{\circ} \mathrm{C}$ and voltage data sets recorded.

The procedure was repeated three times. To identify the changes in fluid conductivity due to acid secretion or other effects, the conductivity of extracted fluids was measured and compared using a HP 4284A impedance analyzer (Hewlett-Packard Instruments).

An ultrasound examination was performed on each of the volunteers to determine the location of the stomach for comparison with EIT images. An experienced ultrasound specialist $(\mathrm{CH})$ reproduced cross-sectional sketches of the abdominal cavity, in the plane of the EIT image from the ultrasound images of the torso for each subject. Ultrasound, rather than MRI, was used for this purpose because the patient could be imaged in a sitting position similar to that for the EIT data collection.

Excessively noisy voltage changes were defined as changes recorded from a single electrode combination which varied by more than $30 \%$ from the mean voltage recorded at the start of each recording under resting conditions. These were removed from data sets and images from the three repeated trials were reconstructed and averaged. The images formed were, in order of observed increasing impedance change; $0.2 \%$ saline referenced to distilled water, $0.9 \%$ referenced to $0.2 \%$ saline, and $0.9 \%$ saline referenced to distilled water.

Percentage changes of impedance in the stomach region of the images were determined from a region of interest surrounding the peak change for each person. Pixels of value greater than half the peak size in the contiguous region of the peak were averaged to calculate the change in impedance for each of the images.

Noise was calculated from the images as the standard deviation of the pixel values in the region outside the stomach, normalized to the percentage change of impedance in the stomach region.

Findings were compared by ANOVA.

\section{RESULTS}

\section{A. Voltage Change as a Function of Reference Phase}

Optimized phases for the demodulator and reciprocity methods were $114^{\circ}$ and $118^{\circ}$, respectively, which corresponded to measured voltage changes of $10.1 \%$ and $10.3 \%$. The expected voltage change, calculated from the measured 


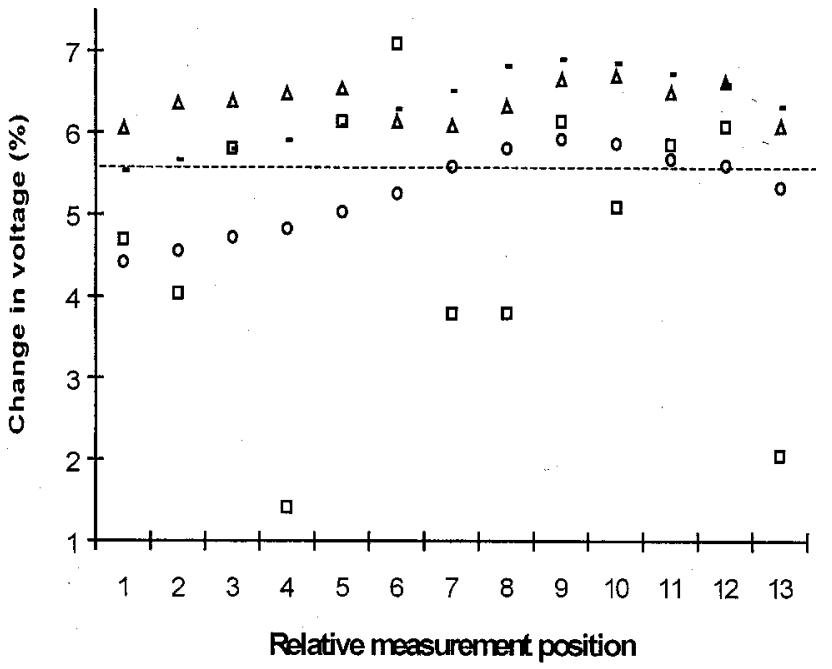

Fig. 2. Graph of the change in voltage versus measurement position on the Cardiff Cole phantom for each of the four optimization techniques, $1 a=$ triangle, $1 b=$ dash, $2 a=$ square, and $2 b=$ circle. The dotted straight line shows the expected change of voltage due to introduction of additional resistance.

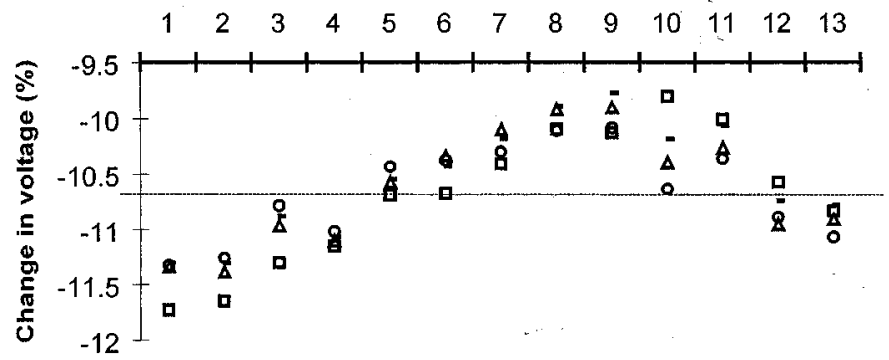

Relative measurement position

Fig. 3. Mean change in voltage versus measurement position on the saline tank, for dilution by $10 \%$, for each of the four optimization techniques, $1 a=$ triangle, $1 b=$ dash, $2 a=$ square, and $2 b=$ circle. The dotted straight line shows the expected change of voltage of $10.7 \%$.

component values, was $10 \%$. The optimized phase determined by the reciprocity method on the circuit with no modeled contact impedance on the voltage measuring channels was $80^{\circ}$, which gave a measured voltage change of $8.3 \%$.

\section{B. Cardiff Cole Phantom}

The residual differences between the expected and measured percentage change for the 13 positions were $2.92 \%, 3.04 \%$, $6.57 \%$, and $2.14 \%$ for techniques $1 \mathrm{a}, 1 \mathrm{~b}, 2 \mathrm{a}$, and $2 \mathrm{~b}$, respectively (Fig. 2). The residual differences and noise for method $2 \mathrm{a}$, the $\mathrm{RE}$ at individual electrodes, was significantly worse $(p<0.001)$ compared with the other techniques. No significant difference was found between the remaining three techniques.

\section{Saline Tank}

1) Changes to Background Resistivity: Residual differences between the expected and measured percentage changes were $1.80 \%, 1.85 \%, 2.15 \%$, and $1.47 \%$ for techniques $1 \mathrm{a}, 1 \mathrm{~b}, 2 \mathrm{a}$, and $2 \mathrm{~b}$, respectively (Fig. 3), when the resistivity of the saline changed by $10.7 \%$. There was no significant difference between

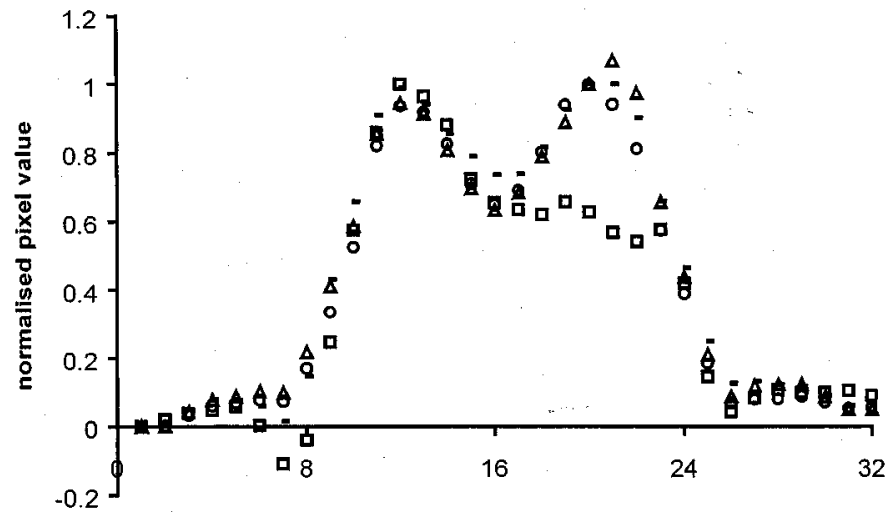

pixel position

Fig. 4. Example of averaged cross-sectional profile through the images of the two sponges separated by $5.6 \mathrm{~cm}$ in the saline-filled tank for the four techniques; $1 a=$ triangle, $1 b=$ dash, $2 a=$ square and $2 b=$ circle.

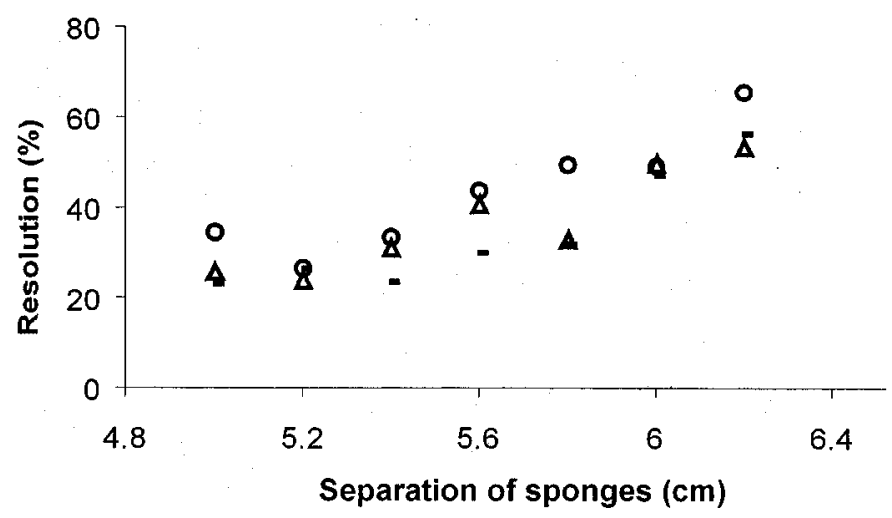

Fig. 5. Resolution versus separation for two sponges in the saline-filled tank for the four techniques; $1 a=$ triangle, $1 b=$ dash, $2 a=$ square and $2 b=$ circle.

the mean changes but method 2a was significantly noisier $(p<$ $0.001)$.

2) Resolution of Two Objects in the Tank: Method $2 \mathrm{~b}$ gave a significantly higher resolution $(p<0.05)$ than methods 1 a and 1b. Method 2a was omitted from the analysis because the profiles for this method were noisy and asymmetric, so that values for resolution could not be calculated (Figs. 4 and 5).

\section{Human Imaging}

Methods $1 \mathrm{~b}$ and $2 \mathrm{~b}$ were most affected by excessively noisy voltage changes. There were $85 \%$ of voltage data sets for method $1 \mathrm{~b}$ that required removal of excessive changes, and $50 \%$ for method $2 \mathrm{~b}$. Methods 1a and 2a both had 5\% of data sets affected.

After removal of noisy channels, there were no significant differences between the sensitivity to impedance change or background noise in images for the four techniques (Figs. 6 and 7).

\section{DisCUSSION}

\section{A. Summary of Results}

The change in voltage measured on the circuit modeling biological impedance variation depended strongly on the phase of 


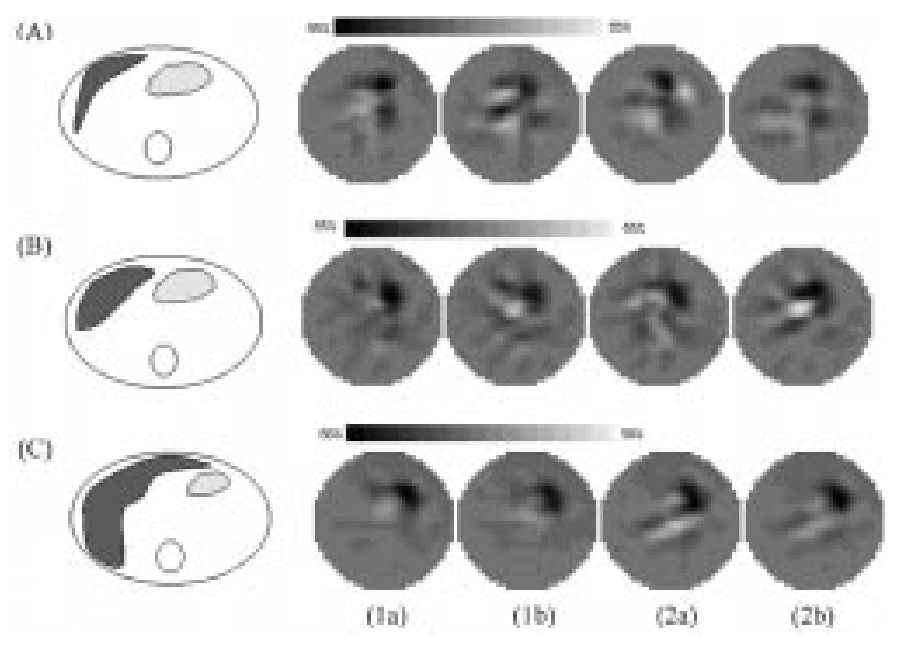

Fig. 6. Example images of the change in impedance between $0.2 \%$ and $0.9 \%$ saline in the stomach of the three volunteers for each of the four techniques. A sketch of the cross section reconstructed from the ultrasound images is included for comparison of the stomach location. For reference the liver and spine are included in the sketch.

the reference at the demodulator. When the mismatch in component values and errors on the drive and receive channels are not large, the two optimization methods gave results within $0.3 \%$ of the expected change in voltage. However, for a large mismatch, the RE was minimized at a very different phase than before, and the corresponding voltage was in error by nearly $20 \%$. On the saline-filled tank and Cardiff Cole phantom experiments, results of the optimization techniques were similar, except for RE at individual electrodes, which gave consistently worse results. The only other difference was that method $2 b$, which used the same phase determined by RE for all electrode combinations, gave better resolution of two objects in a saline-filled tank.

The major difference between the techniques when applied to humans was the number of voltage data sets affected by excessively noisy voltage changes. Methods $1 \mathrm{~b}$ and $2 \mathrm{~b}$, which used the same phases at all electrode combinations, were more affected than methods $1 \mathrm{a}$ and $2 \mathrm{a}$, in which individual electrode combinations were optimized. Sensitivity and background noise in the resulting images were not different between the techniques.

\section{B. Comparison of Individual Optimization and Single Optimization for all Channels}

Due to the variation of errors with electrode combination, it might be expected that a method to optimize phase offset at individual channels would give better results than a method that used the same phase offset for all electrode pairs. This was not apparent with experiments on saline-filled tanks or the Cardiff phantom, but on humans there were significant differences: On humans, the demodulator and reciprocity methods that used individual phases for each electrode combination had far fewer voltage data sets affected by excessively noisy voltage changes compared with the methods that used a single phase for all electrode combinations.

In humans, optimized phase offsets varied much more than in the tanks - by over $100^{\circ}$ from the average calculated for method $1 \mathrm{~b}$, compared with $20^{\circ}$ for a saline-filled tank (Fig. 8). This finding is in agreement with the expectation that an average, or single, reference phase might, therefore, be expected to yield greater errors in human measurements.

\section{Comparison of Reciprocity and Demodulator Techniques}

In all but one of the experiments on saline-filled tanks and the Cardiff Cole phantom, the reciprocity methods gave worse or indistinguishable results to the demodulator methods. On the component network, the only experiment in which the true impedance change was known, the demodulator method also gave superior results. Surprisingly, method 2a, the reciprocity method for individual channels, performed worst on the Cardiff phantom and saline tank. On humans, the demodulator and reciprocity methods optimized for individual electrodes gave indistinguishable results, after excessively noisy channels were eliminated.

The poor performance of method $2 \mathrm{a}$ on the Cardiff phantom and saline tank compared with humans may be attributed to the behavior of RE with phase offset for the different noise conditions. On phantoms and saline tanks, errors due to electrode impedance are small. RE is small for these cases, and did not vary greatly over a large range of phase offsets. Accurate minimization of RE with phase offset is difficult because this function is almost constant with phase and becomes prone to noise. On humans, the mismatch in channel component values due to high and variable skin-contact impedance resulted in a smooth and more minimizable function of RE so that individual reciprocity optimization, unlike in tanks, performed no worse than other methods.

Recommendations: Somewhat surprisingly, there was relatively little difference between the methods. The main difference lay in the number of excessively noisy measurements which were discarded in the human studies, even though the final images were not appreciably different after discarding. The purpose of this study was to arrive at a practical recommendation for a method for use in our clinical studies; the following is mainly based on this difference, plus some more speculative considerations based on other findings in this study.

In humans, optimized phase offsets were sometimes very different from the averaged single optimized phase that is used for all electrode combinations, leading to potentially large errors in calculated voltage changes. On this basis, we recommend that optimization of phase at individual electrode combinations be used in human studies.

Methods based on maximizing the output of the demodulator were more robust to different levels of noise than methods that minimize RE. The RE method gave poor results at low levels of noise, although for the level of noise on humans it appeared to perform relatively well. An advantage of the method that maximizes demodulator output is that only half the number of measurements are needed - it is, therefore, twice as fast as the reciprocity method. At the time of writing, we are using a development of the Mark 1a, the "UCLH Mark 1b system". With 32 electrodes and 258 electrode combinations, optimization with the individual demodulator method takes about $2 \mathrm{~min}$.

We, therefore, recommend that the method that maximizes the output of the demodulator for individual electrodes be adopted. 
(A)

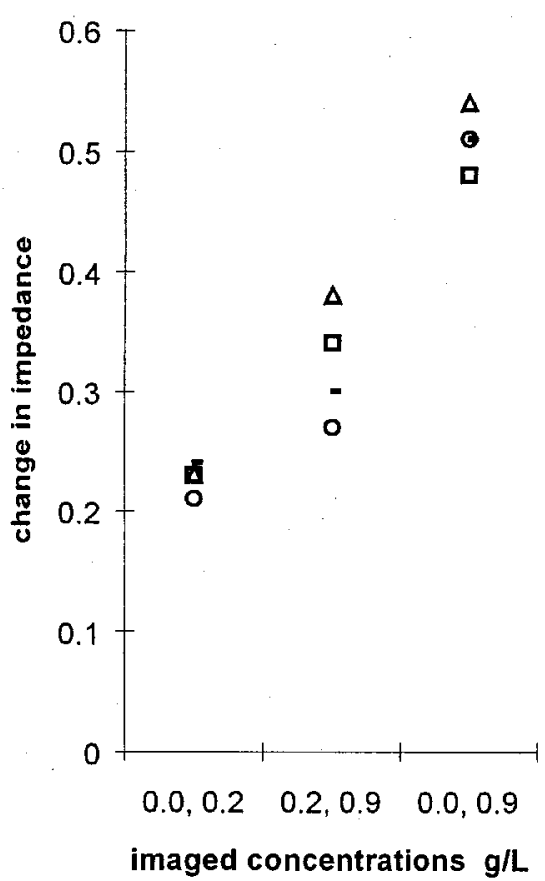

(B)

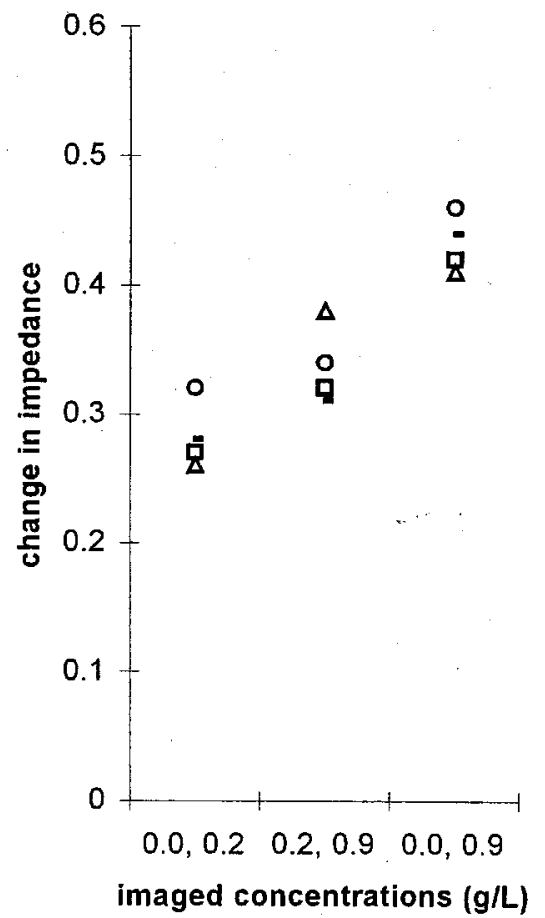

(C)

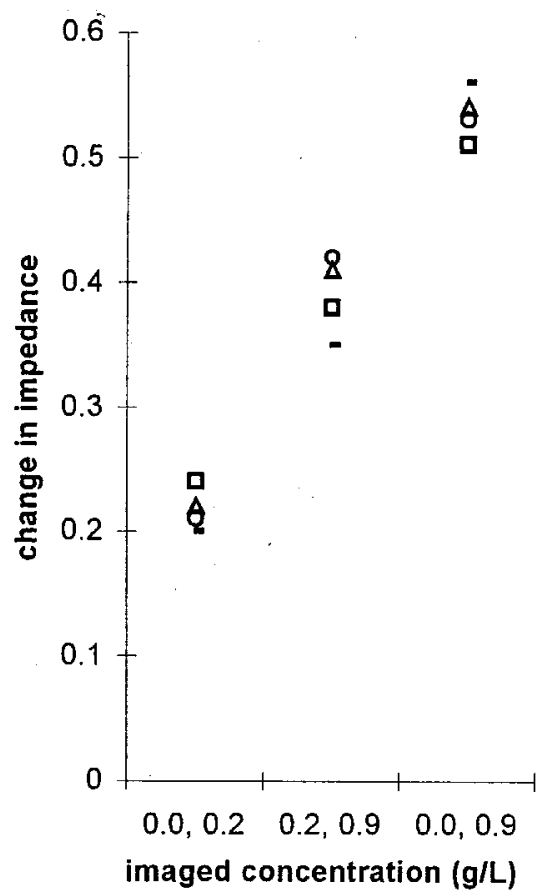

Fig. 7. Comparison of impedance change against saline concentration for each of the three volunteers and the four optimization techniques; $1 a=$ triangle, $1 b=$ dash, $2 a=$ square and $2 b=$ circle.

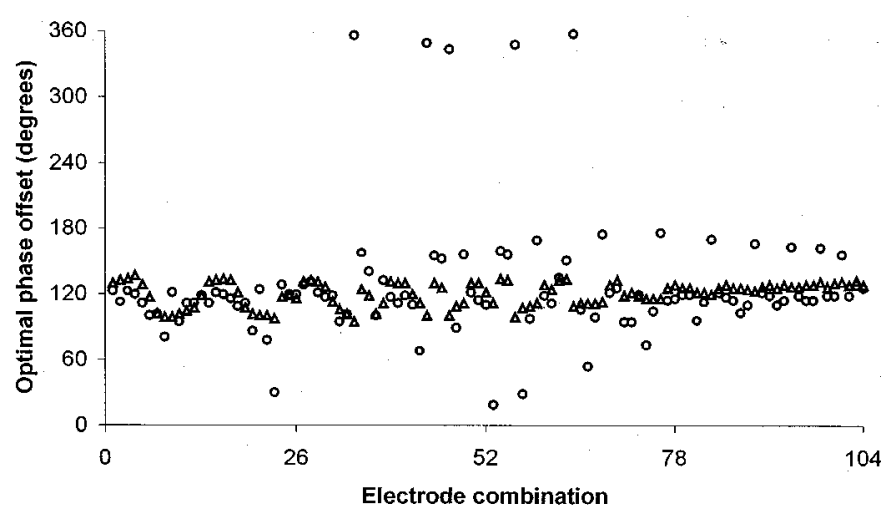

Fig. 8. The change in impedance versus phase shift of the reference (circles) can be fitted by comparing the change in ampitude between two sine waves, one $10 \%$ larger than the other and phase shifted by several degrees (crosses).

\section{Extension to Other EIT Measurements and Designs}

The EIT system used in this study was similar to the Sheffield Mark 1 system, which has been used for the great majority of published clinical studies. The key element to phase optimization is the use of phase-sensitive demodulation, independent of whether this is done in hardware or software. Some systems that use phase-sensitive demodulation do not have the capability of selecting a separate phase offset for each electrode combination. In this case, a phase offset determined by method $1 \mathrm{~b}$, similar to the Sheffield Mark 1 method, would give the fastest and most robust optimization.

The measurements made for this study were all at a frequency of $38 \mathrm{kHz}$. It is unclear which optimization method would be best for other frequencies, as the effects of stray capacitance increase with frequency, but skin-electrode contact impedance decreases. The technique which maximizes the output from the demodulator would also be affected by the deviation from the assumption that the body is dominated by the resistive component.

This study was an empirical one, and was intended to address the practical issue of which method to employ in clinical measurements. The true underlying reasons for the results are complex, and depend on many unknown factors, such as the variation in skin impedance, stray capacitance, and nonidealities in electronic components. We are not aware of any studies that have specifically delineated the errors in EIT measurements by investigating these factors. The recommendations must, therefore, be restricted to the system used, or designs closely similar. It would be desirable to know whether similar findings were applicable to other, more modern, EIT systems, which may measure simultaneously at many frequencies or electrodes, apply voltage rather than current, or use multiple current drive. The use of a constant volume, changing resistivity, test object in the human subjects, is, to our knowledge, novel; the Cardiff phantom was designed as part of an European Community concerted action and several copies were made which can be lent to any interested group.

Although these findings are specific to the system used, all EIT systems suffer from the potential problem that stray capacitance introduces errors in measurement, which renders the choice of phase measurement in the demodulator uncertain. Systems with multiple-electrode current drive, applied voltage or multifrequency recording still need to address this issue. We 
hope that our findings may be generally applicable to other systems, and the methods used here may be suitable for calibration of other systems in this respect.

\section{CONCLUSION}

In this study, using an EIT system with a multiplexer, similar to the Sheffield Mark 1, operating at $38 \mathrm{kHz}$, the most accurate results came from optimizing the phase offset of the reference waveform individually at each individual electrode combination. The method that maximized the output of the demodulator produced less noisy results in human studies than the method that minimized RE, though other measures of accuracy did not vary greatly between optimization methods. The demodulator method also has the advantage that it is twice as fast as the reciprocity method. When optimized individually for each electrode combination, it is, therefore, recommended as the preferable method for the UCLH EIT systems, and those closely similar to it in design.

\section{REFERENCES}

[1] J. G. Webster, Electrical Impedance Tomography. Bristol, U.K.: IOP, 1990.

[2] K. Boone and D. Holder, "Current approaches to analogue instrumentation design in electrical impedance tomography," Phys. Meas., vol. 17, no. 4, pp. 229-247, 1996.

[3] _ "A model of the effect of variations in contact and skin impedance on electrical impedance tomography measurement artefacts," Innov. Tech. Biol. Med., vol. 16, no. 2, pp. 62-70, 1995.
[4] D. Murphy and P. Rolfe, "Aspects of instrumentation design for impedance imaging," Clin. Phys. Physiol. Meas., vol. 9A, pp. 5-14, 1988.

[5] J. Rosell, D. Murphy, R. Pallas, and P. Rolfe, "Analysis and assessment of errors in a parallel data acquisition system for electrical impedance tomography," Clin. Phys. Physiol. Meas., vol. 9A, pp. 93-9, 1988.

[6] P. Riu, J. Rosell, A. Lozano, and R. Pallas-Areny, "Multi-frequency static imaging in electrical impedance tomography: Part 1. Instrumentation requirements," Med. Biol. Eng. Comput., vol. 33, no. 6, pp. 784-792, 1995.

[7] R. Bragos, R. Povill, and P. Riu, "Applicability of the three coefficients calibration technique to EIT," in Abstracts 6th Eur. Community Workshop Electrical Impedance Tomography, Ankara, Turkey, Sept. 16-21, 1994, pp. 49-50.

[8] H. Griffiths, "Modeling of systematic errors in dual-frequency EIT," Innov. Tech. Biol. Med., vol. 15, no. 1, pp. 116-22, 1994.

[9] A. D. Seagar and B. H. Brown, "Limitations in hardware design," Clin. Phys. Physiol. Meas., vol. 8A, pp. 85-90, 1987.

[10] D. B. Geselowitz, "An application of electrocardiographic lead theory to impedance plethysmography," IEEE Trans. Biomed. Eng., vol. BME-18, pp. 38-41, 1971.

[11] B. H. Brown and A. D. Seagar, "The Sheffield data collection system," Clin. Phys. Physiol. Meas., vol. 8A, pp. 91-97, 1987.

[12] H. Griffiths, "A Cole phantom for EIT," Physiol. Meas., vol. 16, pp. 29-38, 1995

[13] H. Griffiths and R. J. Williams, "The Cardiff Cole phantom - A comparison of its theoretical and practical characteristics," Med. Biol. Eng. Comput., vol. 35, no. I, p. 328, 1997.

[14] G. Cusick, D. Holder, A. Birkett, and K. Boone, "A system for impedance imaging epilepsy in ambulatory human subjects," Innov Tech. Biol. Med., vol. 15, no. 1, pp. 33-39, 1994.

[15] D. S. Holder, C. A. Gonzalez-Correa, and T. Tidswell, "Assessment and calibration of a low-frequency system for electrical impedance tomography (EIT), optimized for use in imaging brain function in ambulant human subjects," Ann. NY Acad. Sci., vol. 873, pp. 512-519, 1999.

[16] P. Riu, A. Lozano, M. Fernandez, and R. Pallas-Areny, "Electrode requirements for electrical impedance tomography," in Proc. IVth Int Symp. Biomed. Eng., Peniscola, Spain, 1991, pp. 141-142.

[17] D. S. Holder, Y. Hanquan, and A. Rao, "Some practical biological phantoms for calibrating multifrequency electrical impedance tomography," Physiol Meas., vol. 17, no. 6, pp. 167-77, 1996. 